\title{
Stress Testing Diversified Portfolios: The Case of the CoinShares Gold and Cryptoassets Index
}

\author{
Aikaterini Koutsouri, Michael Petch and William J. Knottenbelt
}

\begin{abstract}
Stress testing involves the use of simulation to assess the resilience of investment portfolios to changes in market regimes and extreme events. The quality of stress testing is a function of the realism of the market models employed, as well as the strategy used to determine the set of simulated scenarios. In this paper, we consider both of these parameters in the context of diversified portfolios, with a focus on the emerging class of cryptoasset-containing portfolios. Our analysis begins with univariate modelling of individual risk factors using ARMA and GJR-GARCH processes. Extreme Value Theory is applied to the tails of the standardised residuals distributions in order to account for extreme outcomes accurately. Next, we consider a family of copulas to represent the dependence structure of the individual risk factors. Finally, we combine the former approaches to generate a number of plausibility-constrained scenarios of interest, and simulate them to obtain a risk profile. We apply our methodology to the CoinShares Gold and Cryptoassets Index, a monthly-rebalanced index which comprises two baskets of risk-weighted assets: one containing gold and one containing cryptoassets. We demonstrate a superior risk-return profile as compared to investments in a traditional market-cap-weighted cryptoasset index.
\end{abstract}

Key words: Cryptoassets, Gold, Index, Risk Modelling, Stress Testing

Aikaterini Koutsouri

Department of Computing, Imperial College London, London, SW7 2AZ, UK

e-mail: k.koutsouri@imperial.ac.uk

Michael Petch

CoinShares, Octagon Point, 5 Cheapside, St Paul's, London, England, EC2V 6AA, UK

e-mail: mpetch@coinshares.co.uk

William J. Knottenbelt

Department of Computing, Imperial College London, London, SW7 2AZ, UK

e-mail: wjk@doc.ic.ac.uk 


\section{Introduction}

Stress testing concepts date back to the early 1990s. During this period, stress testing was mainly used by individual banks for their own risk management plans surrounding their trading activities. The idea of using stress testing was only standardised in 1996 by the Basel Committee on Banking Supervision (BCBS) when an amendment was made to the first Basel Accord (Basel I) [1] that recognised stress testing as an effective way to measure risk. In the second Basel Accord (Basel II) [2], the BCBS asked banks to conduct internal stress testing. However, by the time the financial crisis began in 2007, Basel II was not yet fully implemented internationally.

The financial crisis is an example of a useful stress situation, when banks had to restrict their lending and the limitations of standard value-at-risk methodologies became apparent. At the time, most banks were not properly prepared to accommodate for this situation, which can be linked to the lack of scenario-based risk management planning. Post financial crisis, stress testing has increased widely in implementation across jurisdictions and is used by banks, international organisations, national authorities and academics. Stress tests performed by banks are assessing whether there is enough capital on hand to withstand a potential financial disaster or economic crisis and the results are required to be made publicly available.

In the cryptocurrency industry, while there have been numerous studies of price dynamics $[6,11,8]$, there has been a lack of research in risk management for cryptocurrency-related investment products (e.g. cryptocurrency indices, funds, ETPs, etc.). There is, however, a growing awareness of the importance of this issue in the industry. Additionally, the US Federal Reserve is considering adding to their stress testing framework the scenario of a bitcoin market crash [3]. Nonetheless, there is still plenty of work to be done on the already-existing investment products in the cryptocurrency market by the product owners and potentially market supervisors. On that note, our primary analysis target in this paper is the CoinShares Gold and Cryptoassets Index (CGCI). In the case of the CGCI, we aim to isolate its main design principles and propose a framework for scenario-based risk management that is able to unveil vulnerabilities in certain market conditions.

The remainder of this paper is organised as follows. Section 2 presents the essential background on the principles behind the design of the CGCI and stress testing techniques in practice. In Section 3, we elaborate on the notion of ARMA-GARCH type filtering and how this can be combined with Extreme Value Theory concepts to produce empirical distribution functions of residuals. We further introduce the concept of copulas as an effective mechanism to model dependency structures. Section 3.4 discusses the importance of introducing plausibility constraints in formulated scenarios. Section 4 demonstrates a number of selected stress scenarios for the CGCI that reflects an improved risk profile compared to a traditional market-cap-weighted cryptoasset index. Section 5 concludes. 


\section{Background}

\subsection{Index Methodology}

The CoinShares Gold and Cryptoassets Index (CGCI) [12] is a monthly rebalanced index that employs an inverse volatility weighting scheme for two main components: an equally-weighted basket of five cryptoassets, and physical gold. The main purpose of the CGCI is to provide exposure to the alternative asset space while utilising some means of principled risk control leading to lower volatility. The crypto-basket component of CGCI is composed of the top five eligible cryptoassets based on the 6-month rolling mean of the free-float market capitalisation and their composition is reviewed on a monthly basis when the rebalancing occurs. A more detailed presentation of the index is presented in the full methodology document ${ }^{1}$.

The design of the CGCI is based on two basic premises; the high volatility levels of the cryptoasset space, which brings with it a high level of risk, together with their high intraclass correlation, which limits the diversification benefits. The second is the lack of correlation with physical gold [12]. Based on those characteristics, and Shannon's Demon method of diversification and rebalancing [19], the CGCI utilises the concept of volatility harvesting through (a) forming a basket of cryptoassets and (b) combining it with gold using weighted-risk contribution as a rebalancing mechanism.

Given weighting $x=\left(x_{1}, x_{2}\right)$ for the crypto-basket and gold respectively, implied correlation $\rho=0$, and supposing that we desire the risk contribution of the crypto-basket to be $\alpha$ times the risk contribution of gold, $x_{1}$ is given by:

$$
x_{1}=\frac{\sqrt{\alpha} \sigma_{1}^{-1}}{\sqrt{\alpha} \sigma_{1}^{-1}+\sigma_{2}^{-1}}
$$

Taking into consideration the former, the index is calculated following a two-stage allocation scheme that involves:

1. Computation of the historical volatility of (a) the equally-weighted cryptobasket, and (b) gold;

2. Asset allocation among the crypto-basket and gold expressed as the bivariate weighted risk contribution problem presented in Equation 1. The risk contribution ratio is set as $\alpha=4$, indicating that $80 \%$ of the total risk emanates from the crypto-basket.

The CGCI proposes a mechanism for effective risk control that is sufficient because (a) the cryptoassets class and gold are characterised by respectively high and low levels of volatility respectively, and (b) exhibit insignificant correlation. As such, it is important to consider the effect of changes in both factors.

\footnotetext{
1 To be made available online in due course by CoinShares (UK) Limited
} 


\subsection{Stress Testing}

There are several definitions of stress tests in literature. Studer [21] describes stress tests as the concept of maximum loss over ellipsoidal scenario sets. The stress testing problem is also described by Breuer et al. [4] who propose a number of refinements to Studer's initial approach. According to [4], the quality of the stress test crucially depends on the definition of stress scenarios, which need to meet three requirements: plausibility, severity and suggestiveness of risk-reducing actions. Lopez [14] further refers to stress tests as risk management tools that aim to quantify the impact of unlikely, yet plausible, movements of financial variables on portfolio values.

Stress test design methods vary in practice and are typically divided into two main categories: univariate and multivariate. While univariate stress tests (sensitivity analysis) are easy to perform, they are considered insufficient, as they fail to incorporate the dependence structure of the identified risk factors. Multivariate approaches examine the effects of simultaneous changes in more than one variables and are usually scenario-based. Breuer et al. [4] highlight the importance of an explicit choice of scenarios while Nyström et al. [18] identify the main elements of scenario-based risk management:

1. Recording the market value of the portfolio components

2. Generating scenarios based on a calibrated model of the portfolio behaviour

3. Estimation of the returns distribution

4. Application of risk measures in the obtained distribution

Regarding the model calibrations in step 2, Cherubini et al. [5] consider the bivariate equity portfolio case and suggest a univariate model for each of the two marginal distributions of the portfolio's risk factors (stocks), and one conditional copula approach for the underlying dependence structure.

In the case of CGCI, we identify two main risk factors (the crypto-basket and gold component respectively) and we attempt to model their evolution using stochastic processes. The credibility of results when we apply risk measures to the portfolio distribution, is heavily dependent on the choice of model, and as highlighted by Nyström et al. [18], one should consider a series of stylised facts when simulating the evolution of risk factors. McNeil et al. [15] discuss those stylised facts that characterise the returns of financial time series which can also be observed in the CGCI components.

Stylised facts on autocorrelations and volatility clustering, as proposed by Cont [7], suggest that (i) linear autocorrelations of returns are expected to be very small, (ii) autocorrelation function of absolute returns decays slowly as a function of the time lag and (iii) volatility events are stochastic and appear in clusters. Additionally, we expect (iv) asymmetric and heavy tails in the unconditional distribution of returns that exhibit power-law or Pareto-like behaviors. When examining conditional returns that have been corrected for heteroskedasticity, tails are less heavy. 


\section{Multivariate Stress Testing}

\subsection{Univariate Risk Factor Modelling}

Following the definitions of McNeil et al. [15], the value of a given portfolio at time $t$ can be denoted as $V_{t}$ and modelled as a function of time and a random vector $\boldsymbol{X}_{t}=\left(X_{t, 1}, \ldots, X_{t, d}\right)^{\prime}$, observable at time $t$ and therefore expressed in the form of $V_{t}=f\left(t, \boldsymbol{X}_{t}\right)$, which is typically referred to as mapping of risks. The change in the value of the portfolio will be $\Delta V_{t+1}=V_{t+1}-V_{t}$, the loss is defined as $L_{t+1}:=-\Delta V_{t+1}$ and its distribution is referred to as loss distribution. In this study, we will ultimately be concerned with the distribution of $\Delta V_{t+1}$, termed as the Profit and Loss (P\&L) distribution.

The evolution of each risk factor can be expressed as an autoregressive moving average (ARMA) process, which accounts for autocorrelation and aims to model the conditional mean. Additionally, conditional homoskedasticity is rejected in financial time series, and volatility is stochastic and forecastable. Generalised autoregressive conditional heteroskedasticity (GARCH) processes can adequately account for this fact. Empirical evidence also suggests that positive innovations to volatility correlate with negative market information (and vice versa). Glosten et al. [10] account for this asymmetry through modelling the positive and negative shocks on the conditional variance asymmetrically (GJR-GARCH).

For a combination of the ARMA and GJR-GARCH approaches applied to a time series $X_{t}$, we let $\epsilon_{t}=X_{t}-\mu_{t}=\sigma_{t} Z_{t}$ denote the residuals with respect to the mean process (ARMA error) of order $p_{1}, q_{1}$. We assume that $\sigma_{t}$ follows a $\operatorname{GARCH}\left(p_{2}, q_{2}\right)$ specification, where $p_{2}$ is the order of the squared innovation lag $\left(\epsilon_{t}^{2}\right)$ and $q_{2}$ is the order of the variance lag $\left(\sigma_{t}^{2}\right)$ and finally obtain the asymmetric $\operatorname{ARMA}\left(p_{1}, q_{1}\right)-\operatorname{GARCH}\left(p_{2}, q_{2}\right)$ equations:

$$
\begin{aligned}
X_{t} & =\mu+\sum_{i=1}^{p_{1}} \phi_{i}\left(X_{t-i}-\mu\right)+\sum_{j=1}^{q_{1}} \theta_{j} \epsilon_{t-j}+\epsilon_{t} \\
\sigma_{t}^{2} & =\omega+\sum_{j=1}^{m} \zeta_{j} v_{j t}+\sum_{i=1}^{p_{2}}\left(\alpha_{i} \epsilon_{t-i}^{2}+\gamma_{i} I_{t-i} \epsilon_{t-i}^{2}\right)+\sum_{j=1}^{q_{2}} \beta_{j} \sigma_{t-j}^{2}
\end{aligned}
$$

where $\omega$ is a volatility offset term, $\gamma_{j}$ represents the leverage term and $m$ denotes the number of possible external regressors $v_{j}$. The indicator function $I_{t}$ takes on value of 1 for $\epsilon_{t} \leq 0$ and 0 otherwise.

The main assumption of GARCH models states that standardised residuals are independent and identically distributed (i.i.d.), a key fact to enable us to examine extreme events at the tails of the distributions. In this paper we opt for (a) a Ljung-Box test on standardised residuals to check for evidence of serial autocorrelation, and (b) a Li-Mak test [13] on the standardised residuals to check for remaining ARCH effects. 


\subsection{Tail Behavior Estimates}

Stress scenarios describe how the portfolio would perform under extreme market moves, a fact that yields the modelling process of the risk factors' tails crucial. Extreme Value Theory (EVT) approaches are specifically concerned with the asymptotic behavior of the left and right tails separately. The key assumption of EVT is that it considers i.i.d. sequences. The propagation of asymmetric, heavy-tailed characteristics in the GARCH standardised residuals [22] and their strict white noise behavior, allows for EVT application to the tails. Combined with a non-parametric method for the centre, we can explicitly provide the filtered residual distribution for further simulations.

There are two main approaches that isolate extreme values, block maxima and threshold exceedance models. This is due to the fact that the former study the time series of maxima of consecutive time-series blocks and therefore might disregard large (and often important) portions of the original dataset. In contrast, threshold exceedance methods study all events that exceed a specified high threshold. This study aims to fit a Generalised Pareto Distribution (GPD, the main distributional model for exceedances over threshold) to excess standardised GARCH innovations.

Given a sequence of i.i.d. random values, the Generalised Pareto cumulative distribution function is given by:

$$
G_{\xi, \beta}(x)=\left\{\begin{array}{l}
1-\left(1+\frac{\xi x}{\beta}\right)^{-1 / \xi}, \quad \xi \neq 0 \\
1-e^{-x / \beta}, \quad \xi=0
\end{array}\right.
$$

where $\xi$ and $\beta>0$ denote the shape and scale parameters, $x \geq 0$ when $\xi \geq 0$ and $0 \leq x \leq-\beta / \xi$ when $\xi<0$. Given that $\xi<1$, the GPD mean is $E(X)=\beta /(1-\xi)$. Also, given a random value $X$ with a GPD cumulative distribution function $F=G_{\xi, \beta}$, the cumulative distribution function of the excess distribution over threshold $u$ is given by:

$F_{u}(x)=G_{\xi, \beta(u)}(x)=P(X-u \leq x \mid X>u)=\frac{G_{\xi, \beta(u)}(x+u)-G_{\xi, \beta(u)}(u)}{1-G_{\xi, \beta(u)}(u)}$

where $\beta(u)=\beta+\xi u, 0 \leq x<\infty$ if $\xi \geq 0$ and $0 \leq x \leq-(\beta / \xi)-u$ if $\xi<0$. The excess distribution remains a GPD with the same shape parameter $\xi$ but with a scaling parameter that grows linearly with the threshold parameter $u$. The mean excess function of the GPD is given by:

$$
e(u)=E(X-u \mid X>u)=\frac{\beta(u)}{1-\xi}=\frac{\beta+\xi u}{1-\xi},
$$

where $0 \leq u<\inf$ if $0 \leq u<1$ and $0 \leq u \leq-(\beta / \xi)$ if $\xi<0$. It can be observed that the mean excess function is linear in the threshold $u$, which is a characterising property of the GPD and will assist with the choice of $u$ in later sections. 


\subsection{Dependence Concepts}

In a multivariate approach to risk management one should consider the risk factors' dependence structure. In the case of CGCI, the lack of correlation between the crypto-basket and the gold component is a basic premise that allows for principled risk control through periodic rebalancing. To this end, it is critical to incorporate the dependence structure to the marginal behaviours and investigate the risk sensitivity to different dependence specifications.

In order to quantify the dependence structure, we utilise the concept of copulas, a tool that allows us to decompose a joint probability distribution into its marginals. A $d$-dimensional copula is a distribution function with standard uniform marginal distributions, that represents a mapping of the unit hypercube into the unit interval in the form of $C:[0,1]^{d} \rightarrow[0,1]$. The practicality of copulas is highlighted through Sklar's Theorem that states that any multivariate joint distribution can obtained through (a) the univariate marginal distribution functions and (b) a copula which describes the dependence structure between the variables. Copulas lend themselves particularly useful for this study, where the marginal distributions been defined in detail through the ARMA-GARCH-EVT approach.

For this study we consider two implicit copulas; the Gaussian and t-copula. Given a multivariate normal random vector $\boldsymbol{Y} \sim N_{d}(\boldsymbol{\mu}, \Sigma)$, its copula is a Gaussian copula and, under the property of invariance under monotone increasing transformations, it is the same as the copula of $\boldsymbol{X} \sim N_{d}(\mathbf{0}, P)$, where $P$ is the correlation matrix of $\boldsymbol{Y}$. In two dimensions, the Gaussian copula is given by:

$$
\begin{aligned}
C_{\rho}^{G} & \left(u_{1}, u_{2}\right)=\Phi_{P}\left(\Phi^{-1}\left(u_{1}\right), \Phi^{-1}\left(u_{2}\right)\right) \\
& =\int_{-\infty}^{\Phi^{-1}\left(u_{1}\right)} \int_{-\infty}^{\Phi^{-1}\left(u_{2}\right)} \frac{1}{2 \pi\left(1-\rho^{2}\right)^{1 / 2}} \exp \left(\frac{-\left(s_{1}^{2}-2 \rho s_{1} s_{2}+s_{2}^{2}\right)}{2\left(1-\rho^{2}\right)}\right) d s_{1} d s_{2}
\end{aligned}
$$

where $\Phi$ and $\Phi_{P}$ denote the standard univariate normal distribution function and joint distribution function of $\boldsymbol{X}$ respectively, $P$ is the correlation matrix and $\rho$ is the correlation of $X_{1}, X_{2}$ (unique parameter of $P$ in the bivariate case).

We can similarly define a 2-dimensional t-copula of $\boldsymbol{X} \sim t_{d}(\nu, \mathbf{0}, P)$ by introducing an additional parameter, namely the degrees of freedom:

$$
C_{\rho, \nu}^{t}\left(u_{1}, u_{2}\right)=t_{P, \nu}\left(t_{\nu}^{-1}\left(u_{1}\right), t_{\nu}^{-1}\left(u_{2}\right)\right)
$$

where $t_{\nu}$ and $t_{P, \nu}$ are the standard univariate t distribution function and joint distribution function of $\boldsymbol{X}$ respectively with $\nu$ degrees of freedom, expectation 0 and variance $\frac{\nu}{\nu-2}$, and $P$ is the correlation matrix of $X_{1}, X_{2}$. The degrees of freedom in the t-copula allows to adjust the co-movements of marginal extremes, and that makes t-copulas a popular choice for applications that stress the tail dependencies of risk factors. 
The estimation of the parameters $\boldsymbol{\theta}$ of a parametric copula $C_{\theta}$ is performed through maximum likelihood. If $\hat{F}_{1}, \ldots, \hat{F}_{d}$ denote estimates of the marginal distribution functions, we can construct a so-called pseudo-sample of observations from the copula that consists of the vectors $\hat{\boldsymbol{U}}_{1}, \ldots, \hat{\boldsymbol{U}}_{d}$, where

$$
\hat{\boldsymbol{U}}_{t}=\left(\hat{U}_{t, 1}, \ldots, \hat{U}_{t, d}\right)^{\prime}=\left(\hat{F}_{1}\left(X_{t, 1}\right), \ldots, \hat{F}_{d}\left(X_{t, d}\right)\right)^{\prime} .
$$

The MLE is obtained through maximising:

$$
\ln L\left(\boldsymbol{\theta} ; \hat{\boldsymbol{U}}_{1}, \ldots, \hat{\boldsymbol{U}}_{n}\right)=\sum_{t=1}^{n} \ln c_{\boldsymbol{\theta}}\left(\hat{\boldsymbol{U}}_{t}\right)
$$

with respect to $\boldsymbol{\theta}$, where $\hat{\boldsymbol{U}}_{t}$ denotes the pseudo-observation from the copula and $c_{\boldsymbol{\theta}}$ is the copula density. A goodness-of-fit test can be further used in order to evaluate whether the data is appropriately modelled $[9,20]$.

\subsection{Scenario Plausibility}

The concepts described in the previous sections are combined towards a scenario generation framework that can be summarised in the following steps:

\section{Model Fitting}

1. Define the $d$-dimensional risk factor vector $\boldsymbol{X}_{t}=\left(X_{t, 1}, \ldots, X_{t, d}\right)^{\prime}$, observable at time $t$, and obtain the logarithmic returns time series $\boldsymbol{r}_{t}=\left(r_{t, 1}, \ldots, r_{t, d}\right)^{\prime}$

2. Fit an appropriate asymmetric ARMA-GARCH model to $\boldsymbol{r}_{t}$, and obtain the standardised residuals $\hat{\boldsymbol{Z}}_{t}=\left(\hat{Z}_{t, 1}, \ldots, \hat{Z}_{t, d}\right)^{\prime}$

3. Estimate the marginal distribution functions $F_{1}\left(Z_{1}\right), \ldots, F_{d}\left(Z_{d}\right)$ of the i.i.d. standardised residuals, empirically for the body and with a GPD for the tails

4. Transform $\hat{\boldsymbol{Z}}_{t}$ to uniform variates $\hat{\boldsymbol{U}}_{t}=\left(\hat{U}_{t, 1}, \ldots, \hat{U}_{t, d}\right)^{\prime}$ by inversion

5. Estimate parameters $\boldsymbol{\theta}$ of an appropriate copula $C_{\boldsymbol{\theta}}$ with MLE, given the pseudo-observations $\hat{\boldsymbol{U}}_{t}$

\section{Scenario Generation}

1. For a given sample size $m$, horizon $n$ and parameters $\boldsymbol{\theta}$, simulate $n \times m$ points of the random vector $\boldsymbol{U}=\left(U_{t, 1}, \ldots, U_{t, d}\right)^{\prime}$, with distribution function $C_{\boldsymbol{\theta}}$

2. Given the margins $F_{1}, \ldots, F_{d}$ from step 3 of the fitting process, use quantile transformation to translate to $\boldsymbol{Z}_{t}=\left(F_{1}^{\leftarrow}\left(U_{t, 1}\right), \ldots, F_{d}^{\leftarrow}\left(U_{t, d}\right)\right)^{\prime}$

3. Provide the $n \times m$ standardised innovations matrix pairs to the calibrated ARMA-GARCH models and simulate $m$ paths for each risk factor, $\boldsymbol{X}_{t, \ldots, t+n}$

4. Given the risk factor mapping $V_{t}=f\left(t, \boldsymbol{X}_{t}\right)$ and the simulated returns, construct $m$ portfolio paths and obtain the P\&L distribution 
When examining the credibility of stress test results, plausibility is an important quality criterion that has been studied by existing literature $[4,16]$. The problem setting in those studies consists of a set of elliptically distributed risk factors $\boldsymbol{X}_{t}$, a set of scenarios that represent factor movements, and a linear P\&L function. The Mahalanobis distance is introduced a scenario restriction tool that respects the elliptical symmetry and authors utilise it to define the most likely scenarios given a pre-defined quantile of loss.

In this study, the ultimate goal is to observe variations in the $\mathrm{P} \& \mathrm{~L}$ distribution; we do not examine the intersection of iso-P\&L lines with iso-plausibility ellipses. Nevertheless, we utilise the concept of excluding scenario outliers, as it can bolster the credibility of long-horizon simulations. To this end, preceding step 5 of the described scenario generation procedure, for each set $\boldsymbol{X}_{t, \ldots, t+n}$, we obtain $\mathrm{P} \& \mathrm{~L}$ sets $\mathrm{PL}_{1}, \ldots, \mathrm{PL}_{d}$; we compute the Mahalanobis distance $\mathrm{MD}\left(\mathrm{PL}_{i}\right)$ and filter out samples that exceed a pre-specified percentile of the underlying Chi-Squared distribution of MD. A schematic diagram of the scenario generation procedure in a 2-dimensional risk factor environment is presented in Fig. 1.

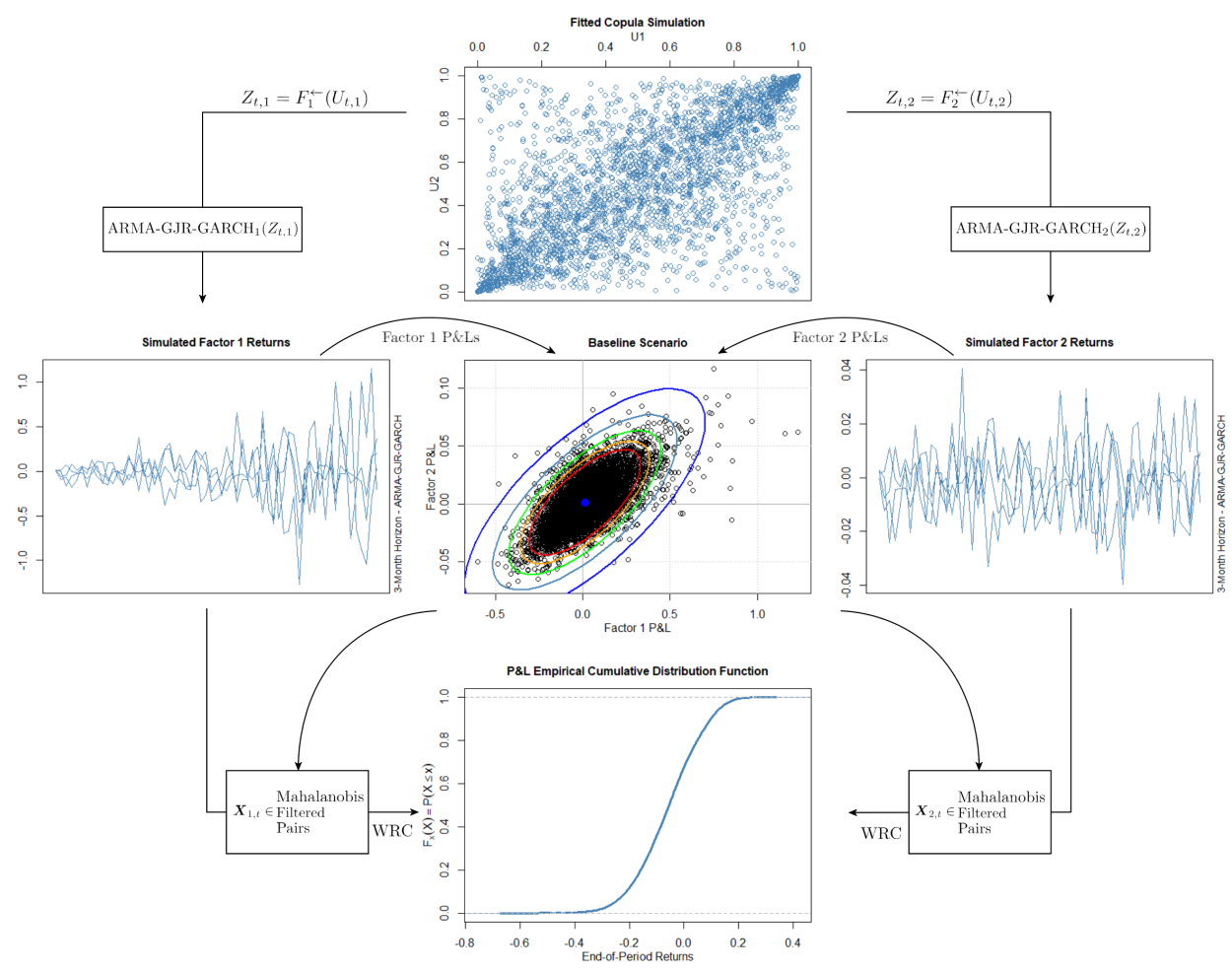

Fig. 1 Scenario generation procedure for 2 risk factors 


\section{Analysis and Results}

\subsection{Index Replication and Risk Mapping}

In the case of CGCI, we isolate two risk factors, namely the crypto-basket and the gold component. The crypto-basket is formulated as an equally-weighted basket of 5 cryptoassets, each with a weight of 0.2 . The crypto-basket price base level is set on $\mathrm{EW}_{0}=100$ on July 1st, 2015.

The crypto-basket price from January 2nd, 2016 onwards is given by:

$$
\mathrm{EW}_{t}=\left(1+\sum_{i \in N_{c, t}} x_{i, R(t)} \times\left(\frac{P_{i, t}}{P_{i, R(t)}}-1\right)\right) \times \mathrm{EW}_{R(t)}
$$

where

- $N_{c, t}$ is the set of the 5 cryptoassets constituents on day $t$

- $R(t)$ is the most recent CGCI rebalancing date preceding $t$

- $P_{i, t}$ is the closing price for cryptoasset $i$ on day $t$, expressed in USD

- $P_{i, R(t)}$ is the closing price for cryptoasset $i$ on the last rebalancing date preceding $t$, expressed in USD

- $x_{i, R(t)}$ is the weight of cryptoasset $i$ on the last rebalancing date preceding $t$, equal to 0.2

- $\mathrm{EW}_{R(t)}$ is the crypto-basket price on the last rebalancing date preceding $t$

The weighting between the crypto-basket and gold in the CGCI is computed through Eq. 1. The Index base level is set on Index $x_{0}=1000$ on January 1st, 2016.

The Index level on day $t$ from January 2nd, 2016 onwards is calculated as:

$$
\operatorname{Index}_{t}=\left(1+\sum_{i \in N_{t}} x_{i, R(t)} \times\left(\frac{P_{i, t}}{P_{i, R(t)}}-1\right)\right) \times \operatorname{Index}_{R(t)}
$$

where

- $N_{t}$ represents the 2 CGCI components (crypto-basket and gold) on day $t$

- $x_{i, R(t)}$ is the weight of constituent $i$ on the last rebalancing date preceding $t$, equal to the WRC allocation result

- Index ${ }_{R(t)}$ is the CGCI price level on the last rebalancing date preceding $t$

We follow Eq. 5 and Eq. 6 and replicate the price time series for the CGCI and transform to logarithmic returns. 


\subsection{Baseline Scenario Simulation}

In this section we simulate a number of scenarios and evaluate the impact of stress in the main assumptions of the CGCI. We differentiate between the baseline, historical and hypothetical scenario. The first simulation describes as realistically as possible a recent index P\&L profile that should serve as a benchmark to evaluate the severity of volatility or correlation shocks. The calibration period is chosen to include all daily observations of 2019 .

The validity of stylised facts presented in Section 2 is verified in Fig. 8-13 in Appendix A for the CGCI components; therefore asymmetric ARMAGARCH processes are likely to explain their evolution. We assume a $t$ distribution for the residuals for the ML process, iterate through pair-wise values of $p \in[1,4], q \in[0,4]$ and choose the combination that yields the minimum AIC. The fitting results are presented in Table 1 of Appendix B.

Next, we estimate the semi-parametric distribution function of the standardised residuals. The linearity of the mean excess function can be used as a diagnostic to assist the selection of appropriate thresholds for the tails. Since the shape and scale parameters in Eq. 4 will be estimated after the threshold is defined, we use an empirical estimator for the mean excess function for positive-valued data $X_{1}, \ldots, X_{n}$, given by:

$$
e^{\mathrm{EMP}}(u)=\frac{\sum_{i=1}^{n}\left(X_{i}-u\right) I_{\left\{X_{i}>u\right\}}}{\sum_{i=1}^{n} I_{\left\{X_{i}>u\right\}}}
$$

We inspect the plot $\left(Z_{i}, e^{\mathrm{EMP}}\left(Z_{i}\right)\right)$, for the positive (Figs. 14 and 16, Appendix A) and for absolute values of the negative innovations (Figs. 15 and 17, Appendix A). For each tail, we define the threshold $u:=Z_{i}$ for such $i$, from which the sample becomes approximately linear for higher values and obtain the parametric GPD tails. The baseline parameters can be found in Table 1. Combined with a Gaussian kernel smoothed interior, we obtain the baseline semi-parametric distribution function, displayed in Figs. 2 and 3.

Given the distribution functions $F_{c}, F_{g}$, we perform inverse transform sampling to the fitted ARMA-GJR-GARCH standardised residuals and fit a copula to the pseudo-sample (Table 1). We proceed with the $t$-copula for the remainder of this paper, as it yields the maximum log-likelihood and provides the means for correlation stress testing through its parameters $(\rho, \nu)$.

With the ARMA-GJR-GARCH parameters, cumulative distribution functions, fitted t-copula for the residuals and a 3-month simulation horizon, we produce 10000 paths for each risk factor, $\boldsymbol{X}_{t, c}, \boldsymbol{X}_{t, q}$. We filter out all path pairs that yield an MD value that exceeds the 99th percentile of the underlying Chi-Squared distribution, and use the remaining to produce $m^{\prime}<m$ paths for the CGCI. Finally, we compute the P\&L at the end of period for each path pair and get the baseline $\mathrm{P} \& \mathrm{~L}$ distribution function. The generated scenarios with the MD plausibility bound, and the final P\&L distribution for the baseline scenario can be found in Figs. 4 and 5 . 


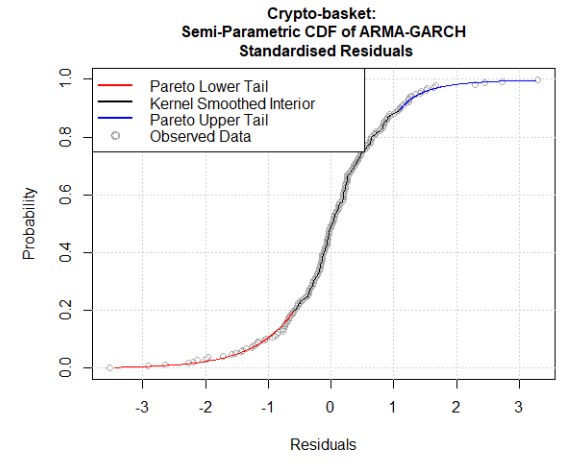

Fig. 2 Semi-parametric CDF Crypto-basket residuals

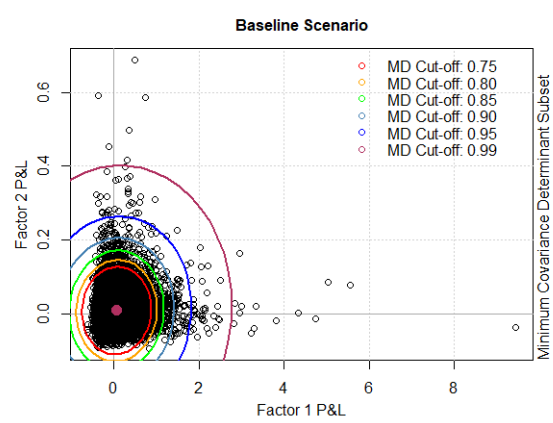

Fig. 4 CGCI baseline scenarios: Mahalanobis plausibility ellipses

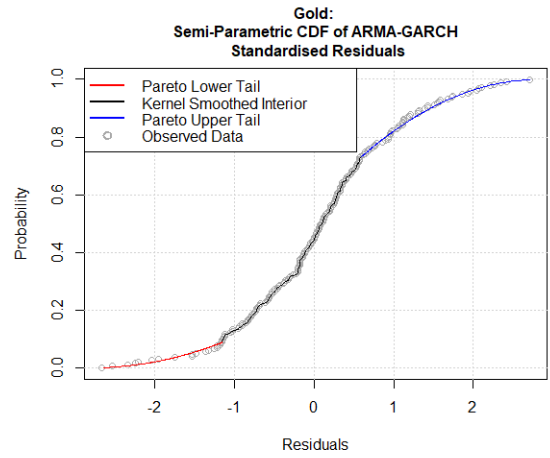

Fig. 3 Semi-parametric CDF Gold residuals

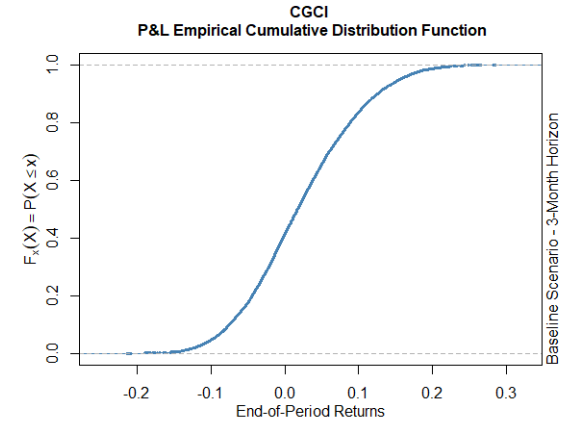

Fig. 5 CGCI baseline scenarios: P\&L distribution

We can also choose to generate a baseline scenario for single risk factor portfolios. In this case, the pseudo-random observations of the residuals can be derived directly from the their cumulative distribution functions. We use this approach to generate 10000 paths of returns for the MVIS Digital Assets 5 Index [17] (MVDA5) - a market capitalisation-weighted index which tracks the performance of the five largest and most liquid cryptoassets - and compare its P\&L distribution with the one derived for the CGCI. The details of the fitting process for the MVDA5 baseline scenario and the plot of the semi-parametric residual distribution can be found in Table 1 of Appendix B and Fig. 18 of Appendix A. 


\subsection{Historical and Hypothetical Scenarios}

The model fitting process can be modified to generate stress scenarios that can be compared against the baseline. First, we change the calibration period to reflect stressful market conditions. Those historical scenarios are commonly used in practice because they are based on events that are observed, and therefore likely to reoccur. For CGCI case, we generate the historical scenarios by calibrating the models in the period of 2018-01-01 to 2018-12-31, as it reflects very large downward price movements in the cryptoasset space.

We use this sample to fit the ARMA-GJR-GARCH model for both risk factors and obtain the i.i.d. standardised residuals. Following the same steps as in Section 4.2, we model the tails and produce the semi-parametric cumulative distribution functions. The new model parameters can be found in Table 1 of Appendix B. We transform the residuals to uniform variates and fit an appropriate t-copula (Table 1 of Appendix B). With the new parameters we generate 10000 paths for each risk factor, filter out implausible pairs, compute the P\&L at the end of the period and obtain the new P\&L distribution that corresponds to the historical scenario. A single-factor approach is also used to produce the MVDA5 historical P\&L distribution. While the historical scenarios can be used to assess the portfolio's performance under realised stressful market conditions, it is biased towards past experience and fails to examine plausible stress events that have not occurred yet.

Additional vulnerabilities are revealed with the generation of hypothetical scenarios. In order to test the resilience to a potential increase in correlation between the CGCI components, we increase the parameter $\rho$ of the t-copula when generating pseudo-samples. We further choose to decrease the degrees of freedom to increase the likelihood of joints extremes. Additionally, we shock the risk factors' volatilities by changing the ARMA-GJR-GARCH volatility constant, $\omega$, and produce volatile risk factor paths. For this study, we modify those parameters such that $\rho=0.9, \nu=2$ and $\omega=10 \omega_{b}$, where $\omega_{b}$ denotes the volatility constant. The P\&Ls that are derived through the modified copula is presented in Fig. 19. The same volatility shock is introduced to the MVDA5 volatility constant to obtain its hypothetical scenario risk profile.

Fig. 6 and Fig. 7 present a comparison of the Baseline, Historical and Hypothetical Scenarios for the case of CGCI and MVDA5. In the case of the CGCI, the slope of the historical P\&L distribution is visibly less steep compared to the baseline. The heavier lower tail further confirms the increased risk in the historical scenario. In Fig. 6 , the probability of a positive return in the historical scenario lies around 31\% compared to $58 \%$ for the CGCI baseline. When it comes to the volatility-shocked scenario, it does not differ significantly around the mid-section, with the probability of profit remaining around $58 \%$, but the CDF is slightly heavier on the upper and lower tail. A single correlation shock impacts the risk profile even less, while a simultaneous shock in both volatility and correlation bring the probability of profit down to approximately $53 \%$. 
In the case of MVDA5, the baseline P\&L distribution reveals significantly higher levels of risk, with no prospect of profit on the 3-month horizon. The historical scenario is more severe, displaying $85 \%$ probability of at least a $25 \%$ loss on the initial investment. The volatility-shocked MVDA5 is heavier on the upper tail, but the probability of a positive end-of-period return barely exceeds 1\%. Overall, CGCI's WRC allocation scheme is characterised by a much more stable risk profile in all the scenarios considered in this study.

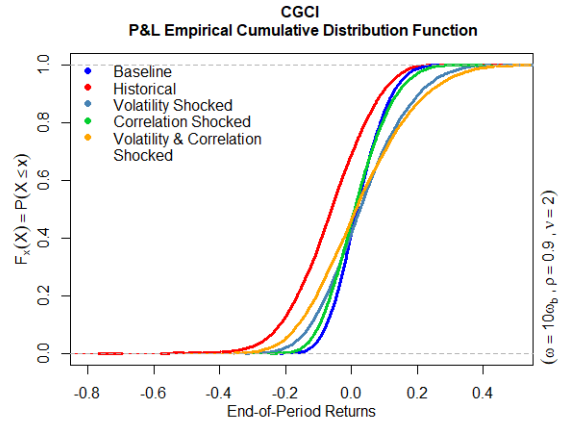

Fig. 6 CGCI P\&L Distributions

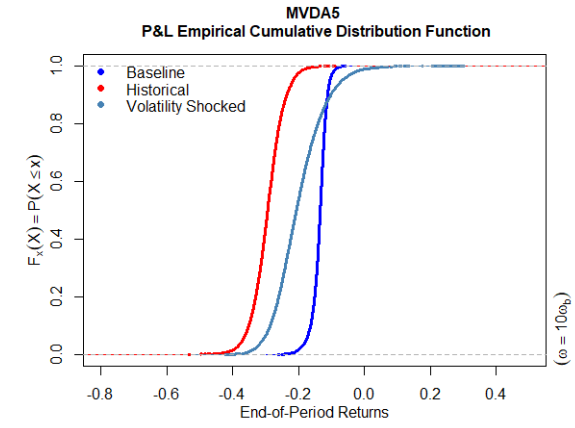

Fig. 7 MVDA5 P\&L Distributions

\section{Conclusion}

We have proposed a framework for scenario-based risk management and have described how it can be applied to assess the risk profile of diversified portfolios with cryptoasset components. The joint evolution of the identified risk factors are modelled in a realistic way and the analysed scenarios are severe, yet plausible. By taking into account a variety of plausible future events related to volatility and correlation levels, we demonstrate the superiority of diversified strategies, such as the CGCI, as a means of mitigating risk. While this application focuses in generating basic stress scenarios specifically for the case CGCI, it can be further modified to include arbitrary combinations of risk factor shocks. Additionally, the presented procedure can potentially be used as a forward-looking portfolio optimisation approach. 


\section{Acknowledgements}

Imperial College of London gratefully acknowledges the support given by CoinShares. Their funding provides Imperial with the support needed to conduct the research found within this paper.

\section{References}

1. Basel Committee on Bank Supervision: Basel I: the Basel Capital Accord (1988)

2. Basel Committee on Bank Supervision: Basel II: International Convergence of Capital Measurement and Capital Standards: a Revised Framework (2004)

3. Board of Governors of the Federal Reserve System: Amendments to policy statement on the scenario design framework for stress testing. Federal Register 84, 6651-6664 (2019)

4. Breuer, T., et al.: How to find plausible, severe, and useful stress scenarios. International Journal of Central Banking 5 (2009)

5. Cherubini, U., et al.: Copula Methods in Finance. John Wiley \& Sons (2004)

6. Chu, J., et al.: GARCH modelling of cryptocurrencies. Journal of Risk and Financial Management 10, 17 (2017)

7. Cont, R.: Empirical properties of asset returns: Stylized facts and statistical issues. Quantitative Finance 1, 223-236 (2002)

8. Elendner, H., et al.: The cross-section of crypto-currencies as financial assets: Investing in crypto-currencies beyond bitcoin. In: Handbook of Blockchain, Digital Finance, and Inclusion, pp. 145-173. Elsevier (2018)

9. Genest, C., Remillard, B.: Validity of the parametric bootstrap for goodness-of-fit testing in semiparametric models. Annales De L'Institut Henri Poincare - Probabilites et Statistiques (2008)

10. Glosten, L.R., et al.: On the relation between the expected value and the volatility of the nominal excess return on stocks. Journal of Finance 48(5), 1779-1801 (1993)

11. Kjærland, F., et al.: An analysis of bitcoin's price dynamics. Journal of Risk and Financial Management 11, 63 (2018)

12. Koutsouri, A., Poli, F., Alfieri, E., Petch, M., Distaso, W., Knottenbelt, W.J.: Balancing cryptoassets and gold: A weighted-risk-contribution index for the alternative asset space. In: Proc. MARBLE 2019. Santorini, Greece (2019)

13. Li, W., Mak, T.: On the squared residual autocorrelations in non-linear time series with conditional heteroscedasticity. Journal of Time Series Analysis pp. 627-636 (2008)

14. Lopez, J.: Stress tests: useful complements to financial risk models. FRBSF Economic Letter (2005)

15. McNeil, A., Frey, R., Embrechts, P.: Quantitative Risk Management: Concepts, Techniques, and Tools, vol. 101. Princeton University Press (2005)

16. Mouy, P., et al.: Extremely (un)likely: a plausibility approach to stress testing. Risk Magazine p. $72-77$ (2017)

17. MV Index Solutions: MVIS CryptoCompare Digital Assets 5 Index. Index Factsheet (2019)

18. Nyström, K., Skoglundy, J.: A framework for scenario-based risk management (2002)

19. Poundstone, W.: Fortune's Formula. Hill and Wang (2005)

20. Remillard, B., et al.: Goodness-of-fit tests for copulas: A review and a power study. Insurance: Mathematics and Economics pp. 199-213 (2009)

21. Studer, G.: Market risk computation for nonlinear portfolios. The Journal of Risk 1(4), 33-53 (1999)

22. Tsay, R.S.: Analysis of Financial Time Series. John Wiley \& Sons (2010) 


\section{Appendix A Plots}
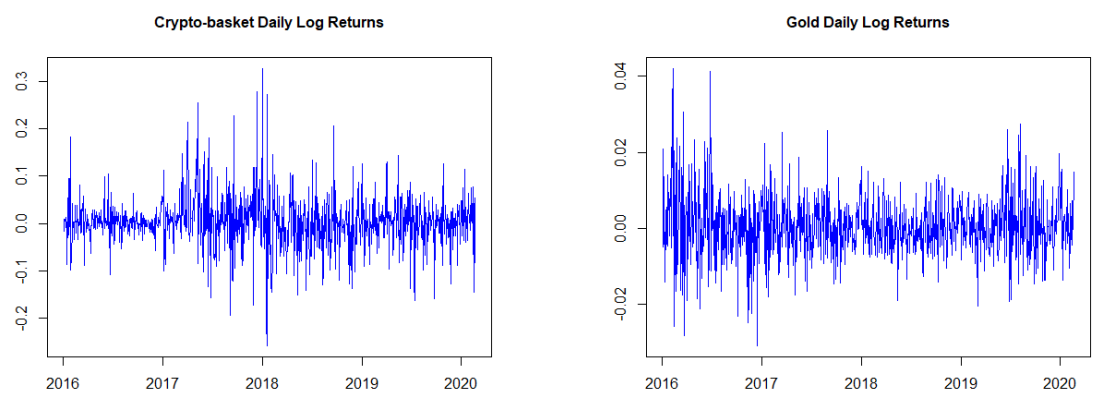

Fig. 8 Crypto-basket log-returns

ACF of Crypto-basket Returns

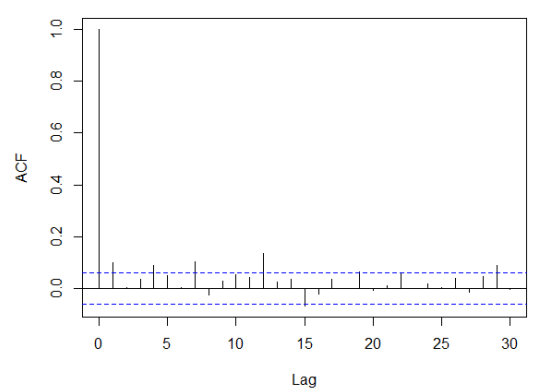

Fig. 10 ACF of Crypto-basket returns

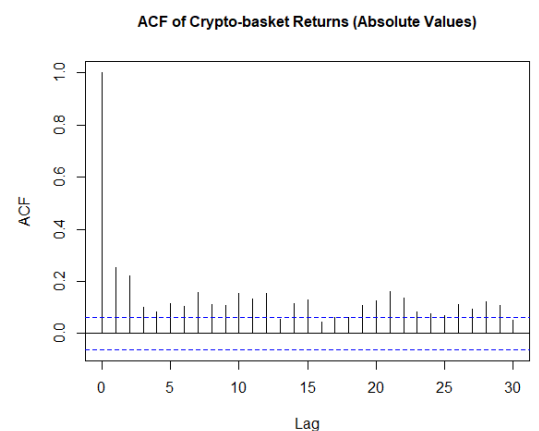

Fig. 12 ACF of Crypto-basket returns (absolute values)

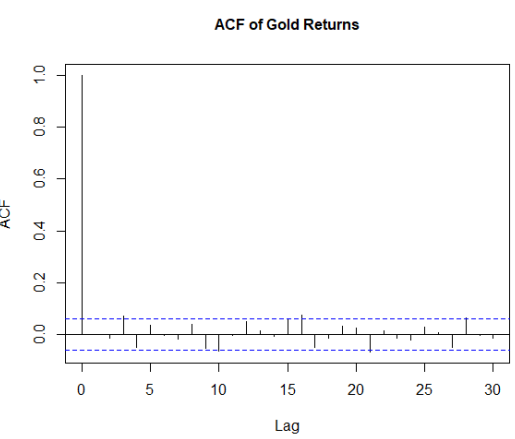

Fig. $11 \mathrm{ACF}$ of Gold returns

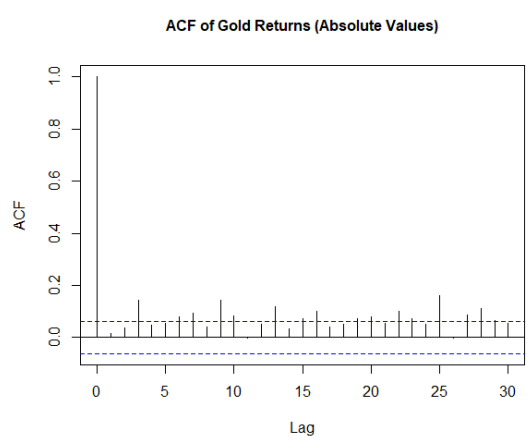

Fig. 13 ACF of Gold returns (absolute values) 

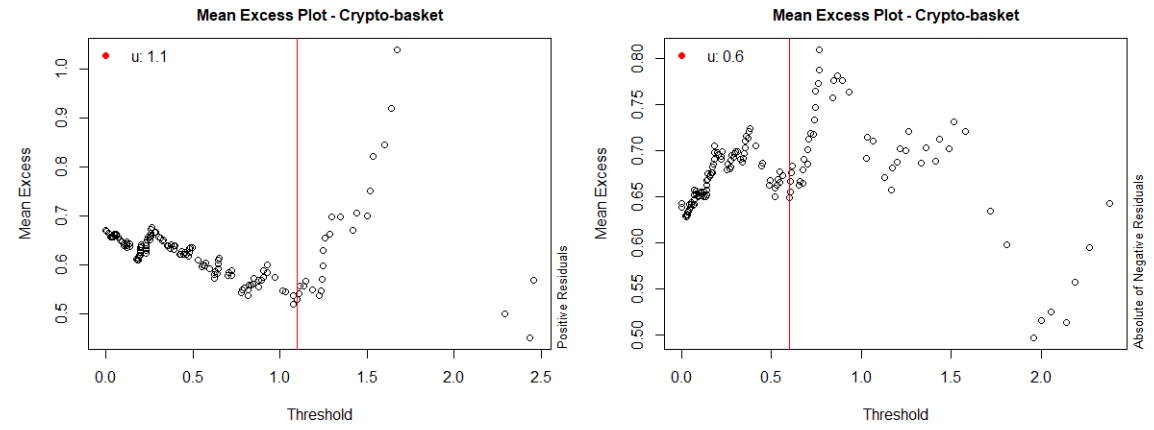

Fig. 14 Crypto-basket mean excess plot Positive residuals

Fig. 15 Crypto-basket mean excess plot Absolute of negative residuals
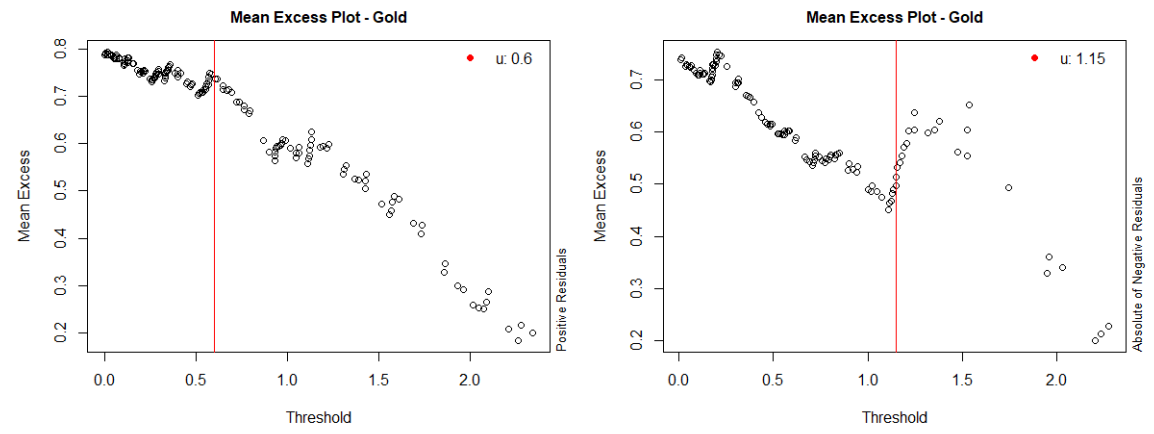

Fig. 16 Gold mean excess plot Positive residuals

Fig. 17 Gold mean excess plot

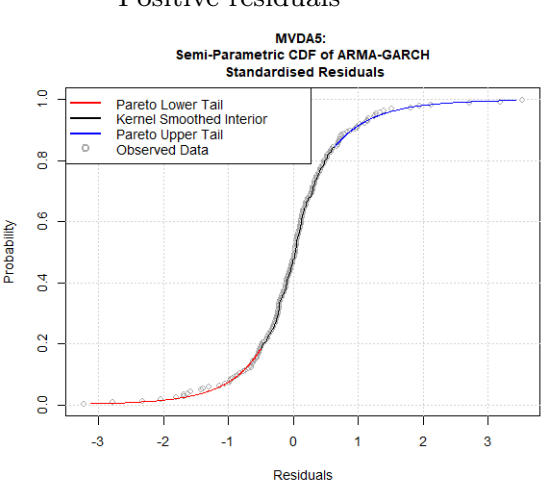
Absolute of negative residuals

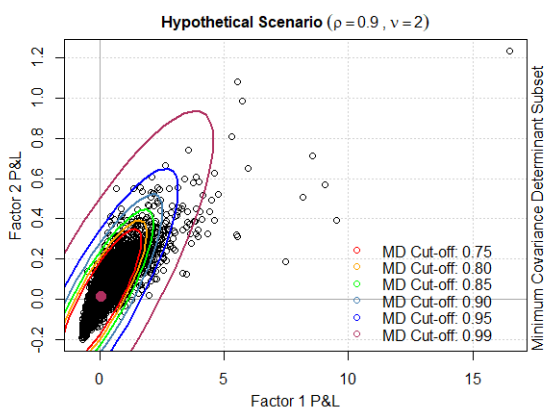

Fig. 18 MVDA5 residuals baseline CDF

Fig. 19 CGCI hypothetical scenarios 


\section{Appendix B Tables}

\begin{tabular}{|c|c|c|c|c|c|c|c|c|c|c|}
\hline \multicolumn{11}{|c|}{ ARMA-GJR-GARCH Fitting } \\
\hline & \multicolumn{2}{|c|}{$\begin{array}{c}\text { Crypto-basket } \\
\text { Baseline }\end{array}$} & \multicolumn{2}{|c|}{$\begin{array}{c}\text { Crypto-basket } \\
\text { Historical }\end{array}$} & \multicolumn{2}{|c|}{$\begin{array}{c}\text { Gold } \\
\text { Baseline }\end{array}$} & $\begin{array}{c}\text { Gold } \\
\text { Historical }\end{array}$ & \multicolumn{2}{|c|}{$\begin{array}{l}\text { MDVA5 } \\
\text { Baseline }\end{array}$} & $\begin{array}{c}\text { MDVA5 } \\
\text { Historical }\end{array}$ \\
\hline $\begin{array}{c}\text { Order } \\
\text { (ARMA) } \\
\text { (GJR-GARCH) }\end{array}$ & & $\begin{array}{l}(4,4) \\
(1,1)\end{array}$ & \multicolumn{2}{|c|}{$\begin{array}{l}(4,4) \\
(1,1)\end{array}$} & \multicolumn{2}{|c|}{$\begin{array}{l}(4,3) \\
(1,1)\end{array}$} & $\begin{array}{l}(4,4) \\
(1,1)\end{array}$ & \multicolumn{2}{|c|}{$\begin{array}{l}(4,2) \\
(1,1)\end{array}$} & $\begin{array}{l}(4,2) \\
(1,1)\end{array}$ \\
\hline $\begin{array}{l}\text { Parameters } \\
\text { (Eq. 2) }\end{array}$ & $\begin{array}{l}\phi_{1}: \\
\phi_{2}: \\
\phi_{3}: \\
\phi_{4}: \\
\theta_{1}: \\
\theta_{2}: \\
\theta_{3}: \\
\theta_{4}: \\
\omega: \\
\alpha_{1}: \\
\gamma_{1}: \\
\beta_{1}:\end{array}$ & $\begin{array}{c}-0.95408 \\
-0.35581 \\
-1.07815 \\
-0.99300 \\
1.03614 \\
0.37295 \\
1.11033 \\
1.06890 \\
0.000007 \\
0.02770 \\
-0.06146 \\
1.00000\end{array}$ & \multicolumn{2}{|c|}{$\begin{array}{c}\phi_{1}: 0.05108 \\
\phi_{2}: 0.45840 \\
\phi_{3}: 0.06855 \\
\phi_{4}:-0.76304 \\
\theta_{1}: 0.08024 \\
\theta_{2}:-0.42010 \\
\theta_{3}:-0.02407 \\
\theta_{4}: 0.92655 \\
\omega: 0.00267 \\
\alpha_{1}: 0.22360 \\
\gamma_{1}: 0.10915 \\
\beta_{1}: 0.13794\end{array}$} & \multicolumn{2}{|c|}{$\begin{array}{l}\phi_{1}:-0.90692 \\
\phi_{2}:-1.00161 \\
\phi_{3}:-0.72425 \\
\phi_{4}: 0.01181 \\
\theta_{1}: 0.79031 \\
\theta_{2}: 1.11737 \\
\theta_{3}: 0.74846 \\
\omega: 0.000001 \\
\alpha_{1}: 0.06469 \\
\gamma_{1}:-0.13793 \\
\beta_{1}: 0.98006\end{array}$} & {$\left[\begin{array}{c}\phi_{1}: 0.59656 \\
\phi_{2}: 1.08368 \\
\phi_{3}:-0.58776 \\
\phi_{4}:-0.39986 \\
\theta_{1}:-0.79959 \\
\theta_{2}:-1.00687 \\
\theta_{3}: 0.76978 \\
\theta_{4}: 0.26998 \\
\omega: 0.00000 \\
\alpha_{1}: 0.00000 \\
\gamma_{1}:-0.00200 \\
\beta_{1}: 1.00000\end{array}\right.$} & \multicolumn{2}{|c|}{$\begin{array}{l}\phi_{1}:-1.21805 \\
\phi_{2}:-1.19354 \\
\phi_{3}:-0.19727 \\
\phi_{4}: 0.02250 \\
\theta_{1}: 1.07132 \\
\theta_{2}: 1.03795 \\
\omega: 0.000002 \\
\alpha_{1}: 0.03245 \\
\gamma_{1}:-0.06707 \\
\beta_{1}: 1.00000\end{array}$} & $\begin{array}{c}\phi_{1}:-1.93720 \\
\phi_{2}:-1.12456 \\
\phi_{3}:-0.12869 \\
\phi_{4}:-0.02543 \\
\theta_{1}: 1.94084 \\
\theta_{2}: 1.03890 \\
\omega: 0.000003 \\
\alpha_{1}: 0.000001 \\
\gamma_{1}: 0.08400 \\
\beta_{1}: 0.94997\end{array}$ \\
\hline AIC & & 3.6341 & \multicolumn{2}{|c|}{-2.7398} & \multicolumn{2}{|c|}{-7.2120} & -7.4216 & \multicolumn{2}{|c|}{-4.0542} & -3.2292 \\
\hline $\begin{array}{c}\text { Residual } \\
\text { Distribution } \\
\text { Shape } \\
\text { (KS Test p-value) }\end{array}$ & \multicolumn{2}{|c|}{$\begin{array}{l}3.32702 \\
(0.7003)\end{array}$} & \multicolumn{2}{|c|}{$\begin{array}{l}3.94689 \\
(0.9604)\end{array}$} & \multicolumn{2}{|c|}{$\begin{array}{c}19.83051 \\
(0.6673)\end{array}$} & $\begin{array}{l}5.93231 \\
(0.8674)\end{array}$ & \multicolumn{2}{|c|}{$\begin{array}{l}2.55280 \\
(0.8684)\end{array}$} & $\begin{array}{l}4.52857 \\
(0.8335)\end{array}$ \\
\hline \multicolumn{11}{|c|}{ Generalised Pareto Distribution Fitting } \\
\hline $\begin{array}{c}\text { Threshold } \boldsymbol{u} \\
\text { (Upper) } \\
\text { (Lower) }\end{array}$ & \multicolumn{2}{|c|}{$\begin{array}{r}1.10114 \\
-0.60602\end{array}$} & \multicolumn{2}{|c|}{$\begin{array}{c}0.54891 \\
-0.75895\end{array}$} & \multicolumn{2}{|c|}{$\begin{array}{c}0.60316 \\
-1.15400\end{array}$} & $\begin{array}{c}0.75454 \\
-0.61262\end{array}$ & \multicolumn{2}{|c|}{$\begin{array}{c}0.63667 \\
-0.51043\end{array}$} & $\begin{array}{c}0.64902 \\
-1.25717\end{array}$ \\
\hline $\begin{array}{c}\text { Parameters } \\
\text { (Upper) }\end{array}$ & \multicolumn{2}{|c|}{$\begin{array}{l}\xi: 0.27178 \\
\beta: 0.39605\end{array}$} & \multicolumn{2}{|c|}{$\begin{array}{l}\xi:-0.03047 \\
\beta: 0.62530\end{array}$} & \multicolumn{2}{|c|}{$\begin{array}{l}\xi:-0.49452 \\
\beta: 1.10766\end{array}$} & $\begin{array}{l}\xi: 0.15642 \\
\beta: 0.53995\end{array}$ & \multicolumn{2}{|c|}{$\begin{array}{l}\xi: 0.09272 \\
\beta: 0.59313\end{array}$} & $\begin{array}{l}\xi:-0.14684 \\
\beta: 0.66304\end{array}$ \\
\hline (Lower) & \multicolumn{2}{|c|}{$\xi: 0.01501$} & \multicolumn{2}{|c|}{$\begin{array}{l}\xi: 0.13459 \\
\beta: 0.57712\end{array}$} & \multicolumn{2}{|c|}{$\begin{array}{l}\xi:-0.46581 \\
\beta: 0.82030\end{array}$} & $\begin{array}{l}\xi:-0.34770 \\
\beta: 1.02221\end{array}$ & \multicolumn{2}{|c|}{$\begin{array}{l}\xi: 0.10358 \\
\beta: 0.52568\end{array}$} & $\begin{array}{l}\xi: 0.28828 \\
\beta: 0.59477\end{array}$ \\
\hline $\begin{array}{l}\text { LogLik } \\
\text { (Upper) } \\
\text { (Lower) }\end{array}$ & \multicolumn{2}{|c|}{$\begin{array}{c}9.66944 \\
30.58602\end{array}$} & \multicolumn{2}{|c|}{$\begin{array}{l}28.99699 \\
28.07471\end{array}$} & \multicolumn{2}{|c|}{$\begin{array}{l}44.36422 \\
8.06560\end{array}$} & $\begin{array}{l}27.00540 \\
40.44401\end{array}$ & \multicolumn{2}{|c|}{$\begin{array}{l}33.08554 \\
203.4029\end{array}$} & $\begin{array}{l}25.21121 \\
27.66995\end{array}$ \\
\hline \multicolumn{11}{|c|}{ CGCI Copula Fitting } \\
\hline & & $\begin{array}{r}\text { Gaussian } \\
\text { Log }\end{array}$ & $\begin{array}{l}\text { Copula } \\
\text { lik }\end{array}$ & $\begin{array}{r}\text { t-Cop } \\
\text { Log }\end{array}$ & pula & t-Cop & $\begin{array}{l}\text { ula Param } \\
(\rho, \nu)\end{array}$ & eters & Good & $\begin{array}{l}\text { dness-of-fit } \\
\text { (p-value) }\end{array}$ \\
\hline Baseline Scen & ario & 0.41 & 348 & 0.611 & 102 & $(0.0$ & $5917,23.624$ & 47) & & 0.7737 \\
\hline Historical Scen & lario & 0.24 & 004 & 0.581 & 172 & $(-0.0$ & $03363,16.62$ & 352) & & 0.3112 \\
\hline
\end{tabular}

Table 1: ARMA-GJR-GARCH, GPD and Copula Parameters and diagnostics 